\title{
Black Hole Masses and Host Galaxy Evolution of Radio-loud Active Galactic Nuclei
}

\author{
Jong-Hak Woo ${ }^{1,2}$, C. Megan Urry ${ }^{3}$, Roeland P. van der Marel ${ }^{4}$, Paulina Lira ${ }^{5}$, Jose Maza ${ }^{6}$
}

\begin{abstract}
We report stellar velocity dispersion measurements for a sample of 28 AGN host galaxies including our previous work. Using the mass-dispersion $\left(M_{\bullet}-\sigma\right)$ and the fundamental plane relations, we estimate the black hole mass for a sample of 66 BL Lac objects and investigate the role of black hole mass in the energetics of BL Lac objects. The black hole mass range for different BL Lac spectral types is similar, $10^{7}<M_{\bullet}<4 \times 10^{9}$. Neither X-ray nor radio luminosity correlates with black hole mass. Low-frequency-peaked BL Lac objects have higher Eddington ratios on average, because of either more beaming or higher intrinsic power. For the black hole mass range $3 \times 10^{7}<M_{\bullet}<10^{9}$, the radio luminosity of BL Lac objects and flat-spectrum radio quasars spans over 4 orders of magnitude, with BL Lac objects being low-power AGNs. We also investigate the evolution of host galaxies for 39 AGNs out to $z \approx 0.5$ with measured stellar velocity dispersions. Comparing the mass-to-light ratio evolution in the observed frame with population synthesis models, we find that single burst star formation models with $z_{\text {form }}=1.4_{-0.2}^{+0.9}$ are consistent with the observations. From our $z_{\text {form }}=1.4$ model, we estimated the intrinsic mass-to-light ratio evolution in the Cousins $R$ band, $\Delta \log (M / L) / \Delta z=-0.502 \pm 0.08$, consistent with that of normal early type galaxies.
\end{abstract}

Subject headings: galaxies: active — galaxies: formation — galaxies: evolution — quasars: general — black hole physics - BL Lacertae objects

\section{Introduction}

The role of black holes in galaxy formation and evolution appears to be significant. There is a tight correlation between black hole mass and the stellar velocity dispersion of bulges in the presentday universe (Ferrarese \& Merritt 2000; Gebhardt

\footnotetext{
${ }^{1}$ Department of Astronomy and Yale Center for Astronomy and Astrophysics, Yale University, P.O. Box 208101, New Haven, CT 06520-8101; jhwoo@astro.yale.edu

${ }^{2}$ Current address: Department of Physics, University of California at Santa Barbara, CA 93106; woo@physics.ucsb.edu

${ }^{3}$ Department of Physics and Yale Center for Astronomy and Astrophysics, Yale University, P.O. Box 208121, New Haven, CT 06520-8121; meg.urry@yale.edu

${ }^{4}$ Space Telescope Science Institute, 3700 San Martin Dr. Baltimore MD 21218; marel@stsci.edu

${ }^{5}$ Departamento de Astronom?a, Universidad de Chile, Casilla 36-D, Santiago, Chile; plira@das.uchile.cl

${ }^{6}$ Departamento de Astronom?a, Universidad de Chile, Casilla 36-D, Santiago, Chile; jmaza@das.uchile.cl
}

et al. 2000), suggesting the growths of black holes and galaxies are closely connected. The rapid decline of star formation rate and quasar activity for the last $\sim 8$ billion years also indicates the co-evolution of black holes and galaxies (Dunlop 1999; Wolf et al 2003). The feedback from Active Galactic Nuclei (AGNs) in star-forming galaxies could quench further star formation and black hole growth at the same time (Silk \& Rees 1998; Wyithe \& Loeb 2003; Springel et al. 2005). To better understand the role of black holes in the AGN-galaxy connection, we are carrying out detailed investigations of active supermassive black holes and the evolution of their host galaxies.

The relation of AGN activity to black hole mass is important to investigate since the black hole mass sets the scale for the gravitational potential and also shows the integral of the accretion history of the black hole. A naive linear scaling between black hole mass and AGN luminosity, ex- 
pected from AGNs accreting at a fixed Eddington ratio, is not observed (Woo \& Urry 2002a; O'Dowd et al. 2002). However, black hole mass estimates with various indirect methods are somewhat uncertain and more accurate data for various types of AGNs at various redshift ranges are needed.

Studies with Hubble Space Telescope (HST $T^{1}$ ) data showed that host galaxies of bright quasars are typically massive ellipticals with a de Vaucouleurs profile (Taylor et al. 1996; Urry et al. 2000; Dunlop et al. 2003), structurally indistinguishable from normal galaxies. Massive earlytype galaxies hosting BL Lac objects and radio galaxies in the local universe $(z<0.1)$ seem to lie on the same fundamental plane as normal galaxies (Falomo et al. 2002; Barth et al. 2003; Woo et al. 2004). Furthermore, Woo et al. (2004) showed that the mass-to-light ratio evolution of AGN host galaxies out to $z \sim 0.3$ is similar to that of normal galaxies, showing that major star formation in the host galaxies occurs earlier than $z \sim 1$, consistent with the mass-to-light ratio evolution of normal early-type galaxies.

To further investigate the relation of AGN activity to black hole mass, the properties of AGN host galaxies, and the relation of active nucleus to host galaxy, we undertook a program of absorption-line spectroscopy for a sample of AGN host galaxies. From stellar velocity dispersions we can infer the black hole mass and study the fundamental relations between black hole mass and other AGN properties. With the addition of morphological information, we can also investigate the evolution of AGN host galaxies using massto-light ratios. First results reported by Woo et al. (2004), who focused on the fundamental plane of host galaxies. Here, we present stellar velocity dispersions, black hole mass estimates, and the mass-to-light ratio evolution for 39 AGN host galaxies (32 BL Lac objects and 7 radio galaxies) out to $z \sim 0.6$, including our previous work. Using the structural parameters of the host galaxies and the fundamental plane relation, we also estimate additional black hole masses for 34 BL Lac objects, to investigate the role of black hole mass in

\footnotetext{
${ }^{1}$ Based on observations made with the NASA/ESA Hubble Space Telescope, obtained from the data archive at the Space Telescope Institute. STScI is operated by the association of Universities for Research in Astronomy, Inc. under the NASA contract NAS 5-26555.
}

the blazar unification paradigm.

In $\S 2$, we describe the observations and velocity dispersion measurements, and in $\S 3$, the AGN black hole mass and its relation with other AGN properties. In $\S 4$, we discuss the evolution of AGN host galaxies, and in $\S 5$ we present the discussion and conclusions. We adopt a cosmology with $\Omega=$ $0.3, \Lambda=0.7$, and $H_{0}=70 \mathrm{~km} \mathrm{sec}^{-1} \mathrm{Mpc}^{-1}$.

\section{Observations and Data Reduction}

We selected $\sim 28$ BL Lac objects and radio galaxies at $z \lesssim 0.6$ with available $H S T$ images to measure stellar velocity dispersions and massto-light ratios. Detailed sample selection and data analysis for the first 15 host galaxies can be found in Woo et al. (2004). Here, we briefly summarize the observations, data reduction, and velocity dispersion measurements for an additional 13 sources at relatively higher redshift.

\subsection{Observations and Data Reduction}

The spectra were obtained with the $\mathrm{B} \& \mathrm{C}$ longslit spectrograph at the $6.5-\mathrm{m}$ Magellan Clay Telescope at Las Campanas Observatory and with the GMOS at the Gemini-South 8-m Telescope. Table 1 shows the details of instrumental setups and the journal of observations. The instrumental setups were chosen to cover strong stellar absorption lines, such as G-band (4300 A), Mgb triplet (around $5172 \AA$ ), and $\mathrm{Ca}+\mathrm{Fe}$ (around $5269 \AA$ ), and to provide sufficient instrumental resolution. Sky conditions were mostly photometric.

The standard data reduction procedures, such as bias subtraction, flat-fielding, spectral extraction, and wavelength calibration, were performed with IRAF routines. One-dimensional spectra were extracted from each exposure and combined to make the final spectrum for the velocity dispersion and redshift measurements.

\subsection{Dispersion Measurements}

We used a direct fitting method, in which the observed spectrum is directly fitted in pixel space with broadened template spectra (van der Marel 1994; Rix et al. 1995; Barth et al. 2002; Woo et al. 2004). The best-fitting dispersion value was determined by minimizing $\chi^{2}$ for the fit. The extracted galaxy and template star spectra were first normalized by a continuum fit. 
The template spectra, taken with each instrument, were convolved with Gaussian velocity profiles, and fitted to the normalized galaxy spectrum using the Gauss-Hermite Pixel Fitting software ${ }^{2}$ (van der Marel 1994). The fitting software uses various polynomial orders and line strength parameters to match galaxy spectra, and determines the best $\chi^{2}$ fit, which gives the velocity dispersion measurement. Galactic absorption lines and various AGN emission lines (e.g., clearly present $\mathrm{H} \beta$ and [OIII] lines) were masked out before fitting.

Extensive and careful fitting in various spectral regions was performed to determine the bestfitting spectral range. Using template stars with different spectral types gives a larger variation in the velocity dispersion. After fitting with each individual and combined template spectrum, we chose the best-template star with the smallest $\chi^{2}$ for each galaxy. Figure 1 shows the host galaxy spectra with the best-fitting broadened templates.

The measured velocity dispersions $(\sigma)$ are corrected for differences in instrumental resolution between the galaxy and template spectra using Eq. 1 in Woo et al. (2004). The instrumental resolution correction changes the velocity dispersion by a few percent. The corrected velocity dispersions and aperture radii for 13 AGN host galaxies are summarized in Table 2.

\subsection{New Redshift Measurements}

We measured the redshift for all observed host galaxies, which include 13 new objects in addition to the 15 reported in Woo et al. (2004). Radial velocity template stars were used to fit overall spectral features including many absorption lines over a wide range of wavelengths. Our high signalto-noise ratio spectra give very accurate redshifts with typical errors less than 0.0001. Values for some redshifts given in the literature turned out to be wrong or inaccurate probably due to lower data quality and sometimes misidentification. In Table 3 we give all updated redshifts for our velocitydispersion-measured AGNs.

We report new redshifts for two BL Lac objects. An uncertain redshift of 0.487 was previously reported for 1248-296 (Padovani \& Giommi $1995)$; its true redshift is $0.3819 \pm 0.0001$ from fitting many absorption lines around the G-band.

\footnotetext{
${ }^{2}$ available at http://www.stsci.edu/ marel/software.html
}

For $1133+161, z=0.46$ was reported by Fichtel et al. (1994) while Rector et al. (2000) estimated a much larger redshift although they reported it as tentative since absorption lines could not be clearly identified in their spectrum. We report $z=0.5735 \pm 0.0001$ for $1133+161$.

\section{AGN Black Holes}

\subsection{Black Hole Mass Estimation}

The reverberation mapping technique gives reliable black hole mass estimates (Peterson 1993), but this method is very expensive, requiring longterm monitoring. An indirect method, using the scaling of the size of the broad-line region with $\mathrm{UV} /$ optical luminosity (Kaspi et al. 2000) is also popularly used for black hole mass estimation. However, this method has a large scatter, limited luminosity range, and in any case can be applied only for broad-line AGNs.

The correlation between black hole mass and galaxy luminosity or stellar velocity dispersion opens a new way of estimating black hole mass for AGNs, including Type II AGNs, radio galaxies, and BL Lac objects. Since the host galaxy magnitude is relatively easy to measure using images with a high quality point spread function, the black hole mass-galaxy luminosity relation can be a powerful tool for estimating black hole mass for a large sample of AGNs. However, scatter in the mass-to-light ratio among galaxies increases the uncertainty. Also, because the black hole massgalaxy luminosity relation is derived from a local galaxy sample, a correction for luminosity evolution of the host galaxies is necessary (Woo et al. 2004), which contributes additional uncertainty.

The black hole mass-stellar velocity dispersion relation is much tighter than the mass-luminosity relation in local galaxies, and it plausibly holds up to an epoch when the bulk of the galaxy mass has assembled, well above the redshift discussed here. Our sample consists of much higher redshift AGNs (out to $z \sim 0.6$ ) than previous samples studied with the mass-dispersion relation (Barth et al. 2002; Falomo et al. 2003; Woo et al. 2004). However, they are still relatively low-redshift AGNs with very massive host galaxies $\left(>10^{11} M_{\odot}\right)$ so it is reasonable to use the mass-dispersion relation; certainly, the mass-dispersion relation for different AGN types and redshift ranges, especially at high 
redshift $(z \gtrsim 1)$ should be investigated further (cf. Treu et al. 2004; Silge et al. 2005).

We use the measured stellar velocity dispersion of host galaxies to estimate the black hole mass via the mass-dispersion relation of Tremaine et al. (2002):

$$
M_{\bullet}=1.349 \times 10^{8} M_{\odot}\left(\sigma_{e} / 200 \mathrm{~km} \mathrm{~s}^{-1}\right)^{4.02},
$$

with proper aperture corrections for $\sigma_{e}$ following Jorgensen et al. (1995). Table 3 presents the black hole masses for our sample of 39 AGNs (which consists of 32 BL Lac objects and 7 radio galaxies; 28 of these we observed ourselves; the remaining 11 have published velocity dispersions from other authors, as summarized in Table 3 of Woo et al. (2004)).

In Figure 2, we compare black hole mass estimates from the measured $\sigma_{e}$ and other, likely less reliable, mass estimates from the calculated $\sigma_{e}$ using $r_{e}$ and $\mu_{e}$ via the Coma cluster fundamental plane. The rms scatter between two black hole mass estimates is 0.34 dex after correcting the luminosity evolution of stellar populations with $\Delta \log L / \Delta \log z=0.502$ in the restframe Cousins R-band from our host galaxy evolution study (see §4.3). Without this evolutionary correction, the black hole masses are systematically higher because of the correlation between the $\log \sigma$ and $\log I_{e}$ in the fundamental plane relation. Although the intrinsic scatter of the fundamental plane and the scatter in the luminosity evolution will increase the uncertainties above those in the mass-dispersion relation, estimating black hole mass from the $r_{e}$ and $\mu_{e}$ could be an alternative way for AGNs with particularly luminous nuclei, where host galaxy stellar velocity dispersions are difficult to measure. In that case, the $K$-correction and the luminosity evolution correction of host galaxies need to be done carefully.

\subsection{The Black Hole Masses and Edding- ton Ratios of BL Lac Objects}

BL Lac objects are AGNs with a relativistic jet oriented toward the line of sight (Urry \& Padovani 1995). Depending on the wavelength of the two broad peaks in their spectral energy distributions (SEDs), they are classified as low-frequencypeaked BL Lac (LBL) or high-frequency-peaked BL Lac (HBL) objects; in particular the classification can be done based on the $\mathrm{X}$-ray to radio flux ratio. ${ }^{3}$ The physical cause of the different SED shapes of BL Lac objects has been the subject of many studies. LBLs from radio surveys are typically more luminous than HBLs from X-ray surveys. This is interpreted as either that LBLs are more beamed (an orientation effect: Ghisellini \& Maraschi 1989; Urry \& Padovani 1995) or that LBLs are intrinsically more luminous (Padovani \& Giommi 1995; Fossati et al. 1997; 1998; Ghisellini et al. 1998).

Here we study the AGN engine for a sample of 32 BL Lac objects with black hole masses from measured stellar velocity dispersions (Table 3 ). In addition, we collected 34 BL Lac objects as a supplementary sample with known redshifts and host galaxy magnitudes (Urry et al. 2000), for which we estimate black hole masses from $r_{e}$ and $\mu_{e}$ via the fundamental plane. X-ray and radio fluxes are collected from the literature using the NED database. ${ }^{4}$

Figure 3 shows black hole mass estimates and the X-ray to radio flux ratio for these samples. The black hole mass of the dispersion measured sample ranges from $\sim 4 \times 10^{7}$ to $\sim 6 \times 10^{8} M_{\odot}$, similar on average to that of radio galaxies and bright quasars, but with a much narrower range (Woo \& Urry 2002a). The lack of higher or lower black hole masses seems to be the result of selection effects. The volume for the dispersion measured sample is too small $(<z>\sim 0.17$ with a standard deviation 0.12 ) to contain the more massive black holes at higher redshifts although we cannot rule out that the black hole mass upper limit of BL Lac objects is much lower than that of other AGNs. When we include the less reliable black hole mass estimates of the supplementary sample, which occupies a larger volume $(\langle z\rangle \sim 0.31$ with a standard deviation 0.17 ), the largest black hole mass increases to $\sim 4 \times 10^{9} M_{\odot}$. Another selection effect is that less massive black holes $\left(\lesssim 10^{7} M_{\odot}\right)$ tend to have fainter host galaxies, which could be below the detection limit of the HST imaging snapshot

\footnotetext{
${ }^{3}$ We use the definition of high (low)-frequency-peaked BL Lac with a dividing line at $\log f_{X} / f_{r}=-5.5$ ( Perlman et al. 1996), with X-ray flux at $1 \mathrm{KeV}$ and radio flux at $5 \mathrm{GHz}$ in Janskys.

${ }^{4}$ The NASA/IPAC Extragalactic Database (NED) is operated by the Jet Propulsion Laboratory, California Institute of Technology, under contract with the National Aeronautics and Space Administration.
} 
survey.

The black hole mass ranges for LBLs and HBLs are similar, as was found previously with much smaller samples (Falomo et al. 2002; Barth et al. 2003; Woo et al. 2004), indicating that black hole mass is not the physical parameter determining BL Lac SED types. Black hole mass also does not correlate with either X-ray or radio luminosity, as shown in Figure 4. LBLs (circles) tends to have higher radio luminosities, almost by definition, but with similar black hole masses as HBLs (triangles). The mean black hole masses of two types are log $\mathrm{M}_{L B L}=8.29 \pm 0.13$ and $\log \mathrm{M}_{H B L}=8.30 \pm 0.06$.

We also calculated bolometric luminosities (without beaming correction) using the radio to bolometric luminosity relation, derived from the blazar sample of Fossati et al. (1998), for which bolometric luminosities were integrated from the SED models as a function of radio luminosity. Figure 5 shows the relation among black hole mass, bolometric luminosity, and Eddington ratio. For a given black hole mass, there are $2-3$ orders of magnitude difference in Eddington ratio, with LBLs generally showing a higher Eddington ratio. This can be interpreted as LBLs have higher apparent Eddington ratio in radio and in bolometric luminosity than HBLs, either because of more beaming or higher intrinsic power (Urry \& Padovani 1995). We cannot differentiate between these two scenarios without an accurate beaming correction which is not possible for individual objects with precision better than an order of magnitude.

\subsection{Radio Power of Blazars}

Since the correlation of black hole mass with radio power was first suggested for a handful of galaxies (Franceschini et al. 1998), several studies have attempted to demonstrate such a correlation (McLure et al. 1999; Lacy et al. 2001; Jarvis \& McLure 2002). However, most of the studied samples seem to suffer from selection effects. Woo \& Urry (2002a, 2000b) showed that the black hole mass ranges are not different between radio-loud and radio-quiet samples with over $400 \mathrm{AGNs.} \mathrm{It}$ has since been shown for a much larger sample of Sloan Digital Sky Survey AGNs $(\sim 6000)$ that for a large black hole mass range $\left(10^{7} \lesssim M_{\bullet} \lesssim\right.$ $\left.10^{10}\right)$, the radio-loudness parameter $\left(F_{5 G H z} / F_{B}\right)$ spans more than 4 orders of magnitude (McLure \& Jarvis 2004, see Figure 2 in their paper), although the mean black hole mass of radio-loud AGNs is a factor of $\sim 1.6$ larger than that of radio-quiet AGNs.

If radio-loud and radio-quiet AGNs are very different populations with different central engines, then a correlation between black hole mass and radio power might exist only among radio-loud AGNs. We compare black hole mass with radio power for our sample of BL Lac objects and for flat-spectrum radio quasars (FSRQs) from Oshlack et al. (2002). The black hole masses of the FSRQs were estimated from the broad-line width and optical luminosity (Oshlack et al. 2002; Woo \& Urry 2000a). If the distribution of BLR clouds is not random and more like a disk distribution, then the velocity of the BLR clouds could be higher by factors of a few (Jarvis \& McLure 2002), and thus the black hole masses larger by as much as an order of magnitude.

Figure 6 shows the black hole mass and radio luminosity at $5 \mathrm{GHz}$ for BL Lac objects (circles and triangles) and FSRQ (crosses). The radio luminosity is not beaming corrected and the intrinsic radio powers of these AGNs are much lower. However, it is clear that radio luminosity between FSRQs and BL Lac objects is different by a minimum of several orders of magnitude for the given black hole mass range. It is unlikely that any beaming correction would reveal a hidden correlation between black hole mass and radio luminosity. Even after increasing the black hole mass of FSRQs by an order of magnitude, considering the possibility of underestimation because of the uncertainties in BLR cloud distribution (Jarvis \& McLure 2002), the radio luminosity still spans over 4 orders of magnitude, indicating no strong correlation between black hole mass and radio power. That is, BL Lac objects and FSRQs may differ in radio luminosity but not in black hole mass, indicating BL Lac objects have lower Eddington ratios than FSRQs. This is consistent with the view that FSRQs and BL Lac objects are the same fundamental class of AGN with different intrinsic radio and line luminosities (Padovani 1992; Maraschi \& Tavecchio 2003). One FSRQ, PKS 0921-213, has relatively low radio power compared with other FSRQ. This quasar was identified as a doublepeaked emission line source, which probably has a considerably lower accretion rate (Eracleous \& Halpern 2003). 


\subsection{Mass - Luminosity Relation for Radio- Loud AGNs}

The Eddington ratio represents how energetic a black hole is for its given mass. Woo \& Urry (2002a) showed that the Eddington ratio spans up to 3 orders of magnitude for given black hole mass of $\sim 300$ AGNs. Here, we revisit the massluminosity correlation of black holes for our sample of BL Lac objects including radio-loud AGNs from Woo \& Urry (2002a).

It is very difficult to determine the Doppler factor for BL Lac jets since superluminal motion and the beaming angle have to be measured for individual objects, from VLBI imaging and constraints from multi-wavelength observations. Good estimates of the Doppler factor do not exist for most of our objects. We instead used an average beaming factor 3.9 for BL Lac objects from Dondi \& Ghisellini (1995), who calculated a lower limit on the Doppler beaming factor for the $\gamma$-ray emission region.

Figure 7 shows bolometric luminosity and black hole mass for radio-loud AGNs including $66 \mathrm{BL}$ Lac objects (circles). It clearly shows that BL Lac objects are intrinsically low-power AGNs with lower Eddington ratios. The Eddington ratio spans at least 4 orders of magnitude, given that the Doppler-factor-corrected luminosity of each BL Lac object is an upper limit. AGN luminosity continuously goes down to non-active levels, however, fainter non-beamed quasars and brighter BL Lac objects do not apper in this plot because of the flux limit and the limited volume, respectively, resulting in a spurious bimodal distribution of bolometric luminosities.

\section{Host Galaxy Evolution}

We studied the evolution of host galaxies for a total sample of 39 radio-loud AGNs: 7 radio galaxies and 32 BL Lac objects. We first measured basic galaxy properties from spectroscopic and imaging data, then computed the mass-to-light ratio for each galaxy and investigated the evolution of the mass-to-light ratio out to $z \sim 0.6$.

\subsection{Host Galaxy Properties}

High signal-to-noise ratio spectra for all our sample galaxies are available (Falomo et al. 2002;
Barth et al. 2003; Woo et al. 2004; this work) and the central stellar velocity dispersions are measured with an aperture corresponding to $3.4^{\prime \prime}$ at the distance of the Coma cluster. The half-light radius, $r_{e}$, and the total galaxy magnitude in the Cousins $R$ band are mainly taken from the HST snapshot survey of BL Lac objects (Urry et al. 2000; see Woo et al. 2004 for other sources). The mean surface brightness within $r_{e}$ is derived from the total magnitude of the host galaxy using:

$<\mu_{e}>=(m-K)+5 \log \left(r_{e}\right)+2.5 \log (2 \pi)-A_{R}-2.5 \log (1+z)^{4}$,

where $m$ is the observed galaxy magnitude, $K$ is the $K$-correction value, $r_{e}$ is the effective radius in arcseconds, and $A_{R}$ is the foreground extinction in the $R$ band taken from the NED database. Initially, we derived the observed-frame $\left\langle\mu_{e}>\right.$ without $K$-correction ( $\S 4.2$ ) and after determining the best-fitting star formation redshift, we calculated the rest-frame $\left\langle\mu_{e}\right\rangle$ with $K$-correction values from our SED models ( $§ 4.3$ ). In Table 2 , we list galaxy properties for the 13 additional sources with new spectroscopic observations presented in this paper.

There are 4 radio galaxies for which $r_{e}$ and total galaxy magnitude measurements from HST data are not available. For 3C 348, we adopted Harris $\mathrm{V}=16.36$ and $r_{e}=22.96^{\prime \prime}$ based on groundbased telescope data (Roche \& Eales 2000). Because the Harris $V$ and the Johnson $V$ have very similar response functions, we convert the $V$ into the Cousins $R$ using $V-R c=0.723$, interpolated for its redshift $z=0.154$, from Fukugita et al. (1995), which is similar to $V-R c=0.721$ from our population synthesis models with $z_{\text {form }}=2$.

For the other three radio galaxies, namely $3 \mathrm{C}$ 135, 3C 424, and 3C 306.1, we could not find any host galaxy magnitude and $r_{e}$ measurements from the literature and thus measured them from $H S T$ images using GALFIT (Peng et al. 2002). PSF images, generated using the Tiny Tim software (Krist \& Hook 1997), were convolved with HST images and the best fit Sersic index and radius were measured. We found that the derived Sersic indices were close to de Vaucouleurs profiles. Hence, we fixed the Sersic index to 4 and derived $r_{e}$ and the total galaxy magnitude. Galaxy properties for the 13 new AGN host galaxies are pre- 
sented in Table 2.

\subsection{Observed Mass-to-Light Ratio Evolu- tion}

From the virial theorem, galaxy masses can be estimated as

$$
\log M=2 \log \sigma+\log r_{e}+C_{1},
$$

where $\sigma$ is in $\mathrm{km} \mathrm{s}^{-1}, r_{e}$ in $K p c, \mathrm{M}$ in $M_{\odot}$ units, and $C_{1}$ is 6.07 (Bender et al. 1992). Log indicates the base 10 logarithm. The average surface brightness within $r_{e}$ is defined by

$$
\log I_{e} \equiv-0.4\left(<\mu_{e}>+C_{o}\right),
$$

in units of $L_{\odot} p c^{-2}$, where $\mu_{e}$ is the average surface brightness within $r_{e}$ in $\operatorname{mag} \operatorname{arcsec}^{-2}$ and $C_{o}=-26.40$ for the Gunn $r$ band (Jorgensen et al. 1996) and -26.05 for the Cousins $\mathrm{R}$ band, taking $R=4.48$ for the Sun (Worthey 1994). Since the luminosity of a galaxy is defined by $L=2 \pi r_{e}^{2} I_{e}$, the mass-to-light ratio can be expressed as

$$
\log M / L=2 \log \sigma-\log I_{e}-\log r_{e}+C_{2},
$$

where the constant $C_{2}=-0.73$ (Jorgensen et al. 1996).

The evolution of $\log M / L$ is the difference in $\log I_{e}$ between two redshift points, assuming the mass, $\sigma$, and $r_{e}$ remain the same. At $z \sim 0, \log I_{e}$ can be derived from the fundamental plane of early type galaxies:

$$
\log I_{e}=\left(b \log r_{e}+c \log \sigma+\gamma\right) / a .
$$

Here, $\mathrm{a}=0.82, \mathrm{~b}=-1, \mathrm{c}=1.24, \gamma=0.182$, as derived for the Coma cluster fundamental plane in the Gunn $r$ band with $r_{e}$ in arcseconds (Jorgensen et al. 1996). If instead $r_{e}$ is expressed in $\mathrm{kpc}$, then $\gamma=-0.120$ if one assumes a Coma distance of $102.9 \mathrm{Mpc}$. After transforming the fundamental plane of the Coma cluster to the Cousins $R$ band, the evolution of the $\mathrm{M} / \mathrm{L}$ ratio in the $R$ band can be written as follows

$\Delta \log M / L=\left(b \log r_{e}+c \log \sigma+\gamma\right) / a+0.4\left(<\mu_{e}\right.$

Here, the Cousins $R$ band surface brightness, $<$ $\mu_{e}(z)>$, is from Eq. 2, $C_{o}=-26.40$ for the Gunn $r$ band, and the color of elliptical galaxies in Coma is $r-R \approx 0.35$ (Jorgensen 1994).
The mass-to-light ratio is usually derived for a rest-frame broad-band magnitude. For high redshift galaxies, it is necessary to apply a $K$ correction to get the rest-frame magnitude from the observed magnitude. The $K$-correction is typically derived from template or model spectra assuming a cosmological model (Fukugita et al. 1995; Poggianti 1997). These models, however, already include an assumption of the galaxy formation epoch. Thus, the $K$-corrected massto-light ratio suffers uncertainties in constraining galaxy formation and evolution. We therefore decided to use the mass-to-light ratio in the observed frame without $K$-correction to constrain the star formation epoch (i.e., we use equations 2 and 7 , but with $K$ set to zero). The advantage of using observed-frame $\mathrm{M} / \mathrm{L}$ ratio evolution is that an a priori assumption on the star formation epoch can be avoided and model predictions with different formation epoch $\left(z_{\text {form }}\right)$ show larger differences in the $\mathrm{M} / \mathrm{L}$ ratio evolution, especially at low redshift.

The evolution of the observed $\mathrm{M} / \mathrm{L}$ ratio for our sample of AGN host galaxies is measured individually and averaged at each redshift bin. We redefine $\Delta \log M / L$ as the difference in logarithm between the $\mathrm{M} / \mathrm{L}$ ratio at a certain redshift and the $\mathrm{M} / \mathrm{L}$ ratio in our lowest redshift bin at $z=0.046$ (Figure 8). So we compare AGNs at different redshift to each other instead of comparing directly to the normal galaxies in Coma. This relative comparison has the advantage that it avoids potential systematic errors resulting from differences in absolute calibration between our AGN dataset and the Coma dataset of Jorgensen et al. (1996). It also removes from the comparison any dependency on the actual distance to Coma. Figure 8 shows that as $\mathrm{z}$ increases, the observed-frame $\mathrm{M} / \mathrm{L}$ remains approximately constant. The stellar populations do get younger and intrinsically brighter. However, this is approximately cancelled by two other effects. First, as $\mathrm{z}$ increases, the observed-frame band corresponds to a bluer restframe band. Since relatively old stellar populations are redder than the Sun, they have lower $R_{t}+L_{\odot} C_{\odot}$ in bluer bands. And second, due to the $(1+z)$ stretching of the spectrum, the observed frame samples a smaller range in wavelength in the rest-frame as $\mathrm{z}$ increases.

We constructed SED models with single burst 
star formation epochs $(z=1-5)$ using Bruzual \& Charlot (2003) models with the Salpeter IMF and the solar metallicity. We then redshifted the models to produce the observed magnitude at each redshift.

Figure 8 shows that the averaged M/L ratio values over each redshift bin (filled circles) is consistent with passive evolution models with $z_{\text {form }}=$ $1-2$. We found that single burst models with $z_{\text {form }}=1.4_{-0.2}^{+0.9}$ best reproduce the observed M/L ratio evolution within the $68 \%$ confidence limit based on $\chi^{2}$ analysis. The last redshift bin has only two points and one of them, 3C 306.1 has clear dust lanes. If we exclude the last bin, then the best fit becomes $z_{\text {form }}=1.4_{-0.2}^{+0.7}$. Therefore, we simply used the $z_{\text {form }}=1.4$ model for further analysis. The derived star formation epoch for our sample of AGN host galaxies is consistent with that of normal galaxies in the field and clusters $\left(z_{\text {form }}=1-3\right.$; van Dokkum \& Franx 2001 ; Treu 2002; Rusin et al. 2003; van Dokkum \& Stanford 2003), indicating normal and AGN host galaxies experience similar formation histories.

\subsection{Intrinsic Mass-to-Light Ratio Evolu- tion}

In order to derive the evolution of the massto-light ratio in the rest-frame $R$ band, we calculated $K$-correction values from our passive evolution model with the determined star formation epoch, $z_{\text {form }}=1.4$. Figure 9 shows the difference in $K$-correction value in our models with different $z_{\text {form }}$ and popularly used models from Fukugita (1995) and Poggianti (1997). The $K$ correction is the difference between the observed magnitude from the redshifted spectrum and the rest-frame magnitude from the de-redshifted spectrum. The $K$-correction value decreases as the formation redshift decreases because stellar populations get younger at a given redshift and thus the observed magnitude decreases. Note that popularly used models predict large $K$-corrections owing to their older adopted cosmology. The $K$ correction can significantly change the intrinsic mass-to-light ratio estimation. At $z=0.5$, the difference in $K$-correction between our $z_{\text {form }}=1.4$ model and the popularly used models corresponds to $\sim 0.05$ in $\log M / L$. We provide $K$-correction values for a couple of single burst stellar population models in Table $4-7$.
With our best-fit $K$-correction value, we derived rest-frame $R$ band magnitudes and the massto-light ratios for individual galaxies. Figure 10 shows the mass-to-light ratio evolution in the restframe Cousins $R$ band for our AGN host galaxies. The averaged mass-to-light ratio indicates $50 \%$ increase in $\mathrm{M} / \mathrm{L}$ ratio between $z=0.05$ and $z=0.4$. which corresponds to $\Delta \log (M / L) / \Delta z=-0.502$ in the Cousins $R$ band and $\Delta \log (M / L) / \Delta z=$ -0.619 in the B band based on our population synthesis model. The lowest and highest $z_{\text {form }}$ models consistent with the data at $68 \%$ confidence, as determined in the previous section $\left(z_{\text {form }}=1.2\right.$ and 2.3 ), indicate a $68 \%$ confidence range for the $\mathrm{M} / \mathrm{L}$ evolution between -0.581 and -0.421 in the Cousins $R$ band, and between -0.695 and -0.517 in the $B$ band. The trend of the M/L evolution of our AGN host galaxies is also similar to that of normal galaxies with $\Delta \log (M / L) / \Delta z=0.46-0.72$ in the $B$ band (Treu et al. 2002; Rusin et al 2003; van de Ven et al. 2003; van Dokkum \& Stanford 2003).

\subsection{Mass vs. $\mathrm{M} / \mathrm{L}$ relation}

We derived the correlation between galaxy mass and mass-to-light ratio (after correcting for luminosity evolution with $\Delta \log (M / L) / \Delta z=-0.502)$ for the AGN host galaxies. The results can be fit with

$\log M / L=(0.45 \pm 0.05) \times \log M-4.39( \pm 0.57)$.

The AGN host galaxies are shown as filled squares in Figure 11, and are compared with nearby earlytype galaxies (open circles) from van der Marel (1991), Magorrian et al. (1998), Kronawitter et al. (2000), and Gebhardt et al. (2003). The mass-tolight ratio of the nearby galaxies are all based on detailed dynamical models for spatially resolved kinematical data (this is more detailed than the values for our own sample, which are based on the virial theorem and an assumption of homology). The compilation into a homogeneous set is from van der Marel \& van Dokkum (2005). All mass-to-light ratio values were transformed to the Cousins $R$ band using $B-R=1.57$ (Fukugita et al. 1995). Distances for the large majority of the nearby galaxies were taken from Tonry (2001) with $H_{0}=70 \mathrm{~km} \mathrm{sec}^{-1} \mathrm{Mpc}^{-1}$. Data for the same galaxies from different authors were averaged. Only galaxies classified as ellipticals are in- 
cluded in the nearby galaxy sample. The few most distant galaxies from Magorrian et al. (1998) were removed from the sample because their models for these galaxies included unrealistically large black hole masses. We find that normal and AGN host galaxies have a similar relation between mass and $\mathrm{M} / \mathrm{L}$ ratio in the galaxy mass range where the data sets overlap, $11<\log M / M_{\odot}<12$. The most massive galaxies in the AGN sample suggest the possibility of a break in the $M / L$ vs. $M$ relationship at masses in excess of $10^{12} M_{\odot}$.

\section{Discussion and Conclusions}

We measured the stellar velocity dispersions for 21 BL Lac object host galaxies and 7 radio galaxies from our spectroscopic observations. Including 11 velocity-dispersion-measured BL Lac object host galaxies from the literature, we estimated black hole masses for a sample of 39 AGN host galaxies using the mass-dispersion relation. We also estimated black hole masses for 34 additional BL Lac objects using the derived velocity dispersion from well-measured $r_{e}$ and $\mu_{e}$ of the host galaxies and the fundamental plane relation.

Estimating black hole mass from $r_{e}$ and $\mu_{e}$ seems promising since the intrinsic scatter in the fundamental plane is small. Although highresolution imaging is required for an accurate AGN subtraction to derive reliable host galaxy properties, it would be more feasible than longexposure spectroscopy in the case of typical quasars, of which a featureless AGN continuum is much brighter than host galaxy absorption features.

The black hole mass of BL Lac objects ranges from $10^{7}$ to $4 \times 10^{9} M_{\odot}$. We found no strong correlation between black hole mass and either X-ray or radio luminosity. HBLs and LBLs have similar black hole masses but LBLs show higher Eddington ratios in radio and bolometric luminosity, because of either more beaming or higher intrinsic power. We also compared FSRQs and BL Lac objects and found that their black hole masses are similar but their radio luminosities are quite different, indicating that BL Lac objects and FSRQs are plausibly the same objects with different Eddington ratios as suggested by other blazar unification study (Maraschi \& Tavecchio 2003).

All black hole mass estimates depend on the mass-dispersion relation observed in the presentday universe. A recent study on 7 Seyfert 1 galaxies at $z \sim 0.4$ suggests an evolution of the massdispersion relation with a higher black hole mass for a given velocity dispersion (Treu et al. 2004). Neglecting the black hole mass growth for the last 4 billion years, this could indicate a mass evolution of spheroids. If this is the case, then our black hole mass estimates are a lower limit. However, the host galaxies in our sample are very massive elliptical galaxies, consistent with pure luminosity evolution since $z \sim 1$, and probably represent a different population than Seyfert 1 galaxies.

We measured the mass, mass-to-light ratio, and the evolution of the mass-to-light ratio for the sample of 39 AGN host galaxies. From the observed-frame (no $K$-correction) mass-to-light ratio evolution, we tested single burst star formation epoch models using our population synthesis models. The passive evolution model with $z_{\text {form }}=1.4_{-0.2}^{+0.9}$ is consistent with the observed mass-to-light ratio evolution. From a passive evolution model with $z_{\text {form }}=1.4$, we measured the evolution of the intrinsic mass-to-light ratio in the Cousins $R$ band, which is $\Delta \log (M / L) / \Delta z=$ $-0.502 \pm 0.08$. The mass-to-light ratio evolution of our sample of AGN host galaxies is similar to that of normal galaxies, indicating that normal and AGN host galaxies experience similar star formation histories. Whether the supermassive black hole is active at the observed epoch seems not related to the global star formation history. However, we note that our host galaxies are among the most massive galaxies $\left(>10^{11} M_{\odot}\right)$ and the star formation redshift is marginally lower than that of normal galaxies with the same mass range $\left(z_{\text {form }} \gtrsim 2-3\right.$; Treu et al. 2005 ; van der Wel et al. 2005) indicating 1-2 Gyr younger age, which implies either a later epoch of star formation or additional star formation in AGN host galaxies. Rest-frame colors of host galaxies can shed light on more detailed interpretation.

In contrast to host galaxies at high redshift $(z \gtrsim 2)$, when galaxies and black holes are still assembling their masses, early-type host galaxies at low redshift $(z<1)$ are grown-up galaxies with a typical mass $\gtrsim 10^{11} M_{\odot}$. These host galaxies seem just like normal galaxies except for their active central black holes, which are probably revived from dormant status. In the case of late-type 
host galaxies, where star formation and AGN activity can be more closely connected, host galaxies might show very different properties compared to normal galaxies. Further investigation of the relation of nuclear activity to host galaxy properties for AGNs at higher redshift and with lower host galaxy mass is required to understand the full picture.

This research is a part of the AGN Key Project of the Yale-Calan collaboration, and has been supported by Fundacion Andes. Partial support was also provided by NASA grant AST-0407295. Partly based on observations obtained at the Gemini Observatory, which is operated by the Association of Universities for Research in Astronomy, Inc., under a cooperative agreement with the NSF on behalf of the Gemini partnership: the National Science Foundation (United States), the Particle Physics and Astronomy Research Council (United Kingdom), the National Research Council (Canada), CONICYT (Chile), the Australian Research Council (Australia), CNPq (Brazil) and CONICET (Argentina).

\section{REFERENCES}

Barth, A., Ho, L., \& Sargent, W. L. W. 2002, ApJ, 566, L13

Bender, R., Burstein, D., \& Faber, S. M. 1992, ApJ, 399, 462

Bruzual, G., \& Charlot, S. 2003, MNRAS, 344, 1000

Dondi, L., \& Ghisellini, G. 1995, MNRAS, 273, 583

Dunlop, J. 1999, in The Most Distant Radio Galaxies, ed. H. J. A. Röttgering, P. N. Best \& M. D. Lehnert (Dordrecht: Kluwer), p. 71

Dunlop, J. S., McLure, R. J., Kukula, M. J., Baum, S. A., \& Hughes, D. H. 2003, MNRAS, 340, 1095

Eracleous, M., \& Halpern, J. P. 2003, ApJ, 599, 886

Falomo, R., Kotilainen, J. K., Carangelo, N., \& Treves, A. 2003, ApJ, 595, 624

Ferrarese, L., \& Merritt, D. 2000, ApJ, 539, L9
Fichtel et al. 1994 ApJS, 94,551

Fossati, G., Maraschi, L., Celotti, A., Comastri, A. \& Ghisellini, G. 1998, MNRAS, 299, 433

Franceschini, A., Vercellone, S., \& Fabian, A. C. 1998, MNRAS, 297, 817

Fukugita, M., Shimasaku, K., \& Ichikawa, T. 1995, PASP, 107, 945

Gebhardt, K., Bender, R., Bower, G., et al. 2000a, ApJ, 539, 13

Gebhardt, K., et al. 2003, ApJ, 583, 92

Ghisellini, G., \& Maraschi, L. 1989, ApJ, 340, 181

Ghisellini, G., Celotti, A., Fossati, G., Maraschi, L., \& Comastri, A. 1998, MNRAS, 301, 451

Jarvis, M. J. \& McLure, R. J. 2002, MNRAS, 336, L38

Jorgensen, I. 1994, PASP, 106, 967

Jorgensen, I., Franx, M., \& Kjaergaard, P. 1995, MNRAS, 276, 1341

Jorgensen, I., Franx, M., \& Kjaergaard, P. 1996, MNRAS, 280, 167

Kaspi, S., et al. 2000, ApJ, 533, 631

Krist, J. E., \& Hook, R. N. 1997, The 1997 HST Calibration Workshop with a New Generation of Instruments, p. 192

Kronawitter, A., Saglia, R. P., Gerhard, O., \& Bender, R. 2000, A\&AS, 114, 53

Lacy, M., Laurent-Meuleisen, S. A., Ridgway, S. E., Becker, R. H., \& White, R. L. 2001, ApJ, 551, L17

Maraschi, L. \& Tavecchio, F. 2003, ApJ, 593, 667

Magorrian, J., et al. 1998, AJ, 115, 2285

McLure, R. J., \& Jarvis, M. J. 2004, MNRAS, 353, 45

McLure, R. J., Kukula, M. J., Dunlop, J. S., Baum, S. A., \& O'Dea, C. P. 1999, MNRAS, 308,377

O’Dowd, M., Urry, C. M., \& Scarpa, R. 2002, ApJ, 580,96 
Oshlack, A., Webster, R., \& Whiting, M. 2002, ApJ, 575, 810

Padovani, P. 1992, MNRAS, 257, 404

Padovani, P., \& Giommi, P. 1995, MNRAS, 277, 1477

Peng, C., et al. 2002, AJ, 124, 266

Perlman, E. S., Stocke, J. T., \& Wang, Q. D. 1996, ApJ, 456, 451

Peterson, B. M. 1993, PASP, 105,207

Poggianti, B. M. 1997, A\&AS, 122, 399

Rix, H. W. R., Kennicutt, R. C., Jr., Braun, R., \& Walterbos, R. A. M. 1995, ApJ, 438, 155

Rector, T. A., et al. 2000, AJ, 120, 1626

Roche, N., \& Eales, S. A. 2000, MNRAS, 317, 120

Rusin, D., et al. 2003, ApJ, 587, 143

Silge, J. D., et al. 2005, AJ, submitted (astro$\mathrm{ph} / 0501446$ )

Silk, J. \& Rees, M. J. 1998, AA, 331, L1

Springel, V., Di Matteo, T., \& Hernquist, L. 2005, ApJ, 620, L79

Taylor, G. L., Dunlop, J. S., Hughes, D. H., \& Robson, E. I. 1996, MNRAS, 283, 930

Tonry, J. L., et al. 2001, ApJ, 546, 681

Tremaine, S., et al. 2002, ApJ, 574, 740

Treu, T., Stiavelli, M., Casertano, S., Mller, P., \& Bertin, G. 2002, ApJ, 564, L13

Treu, T., Malkan, M., \& Blanford, R. D. 2004, ApJ, 615, L97

Treu, T., Ellis, R. S., Liao, T. X., \& van Dokkum, P. G. 2005, ApJ, 622, 5

Urry, C. M., Scarpa, R., O'Dowd, M., Falomo, R., Pesce, J. E., \& Treves, A. 2000, ApJ, 532, 816

Urry, C. M. \& Paolo, P. 1995, PASA, 107, 803

van der Marel, R. P. 1991, MNRAS, 253, 710

van der Marel, R. P. 1994, MNRAS, 270, 271 van der Marel, R. P. \& van Dokkum, P. G. 2005, ApJ, sumitted

van der Wel, P. M., et al. 2005, ApJ, submitted

van de Ven, P. M., van Dokkum, P. G., \& Franx, M. 2003, MNRAS, 344, 924

van Dokkum, P. G., \& Franx, M. 2001, ApJ, 553, 90

van Dokkum, P. G., \& Stanford, S. A. 2003, ApJ, 585,78

Worthey, G. 1994, ApJS, 95, 107

Wyithe, J. S. B., \& Loeb, A. 2003, ApJ, 595, 614

Wolf, C., Meisenheimer, K., Rix, H.-W., Borch, A., Dye, S., \& Kleinheinrich, M. 2003, A\&A, 401, 73

Woo, J.-H., \& Urry, C. M. 2002a, ApJ, 579, 530

Woo, J.-H., \& Urry, C. M. 2002b, ApJ, 581, L5

Woo, J.-H., Urry, C. M., Lira, P., van der Marel, R., \& Maza, J. 2004, ApJ, 617, 903

This 2-column preprint was prepared with the AAS LATEX macros v5.2. 

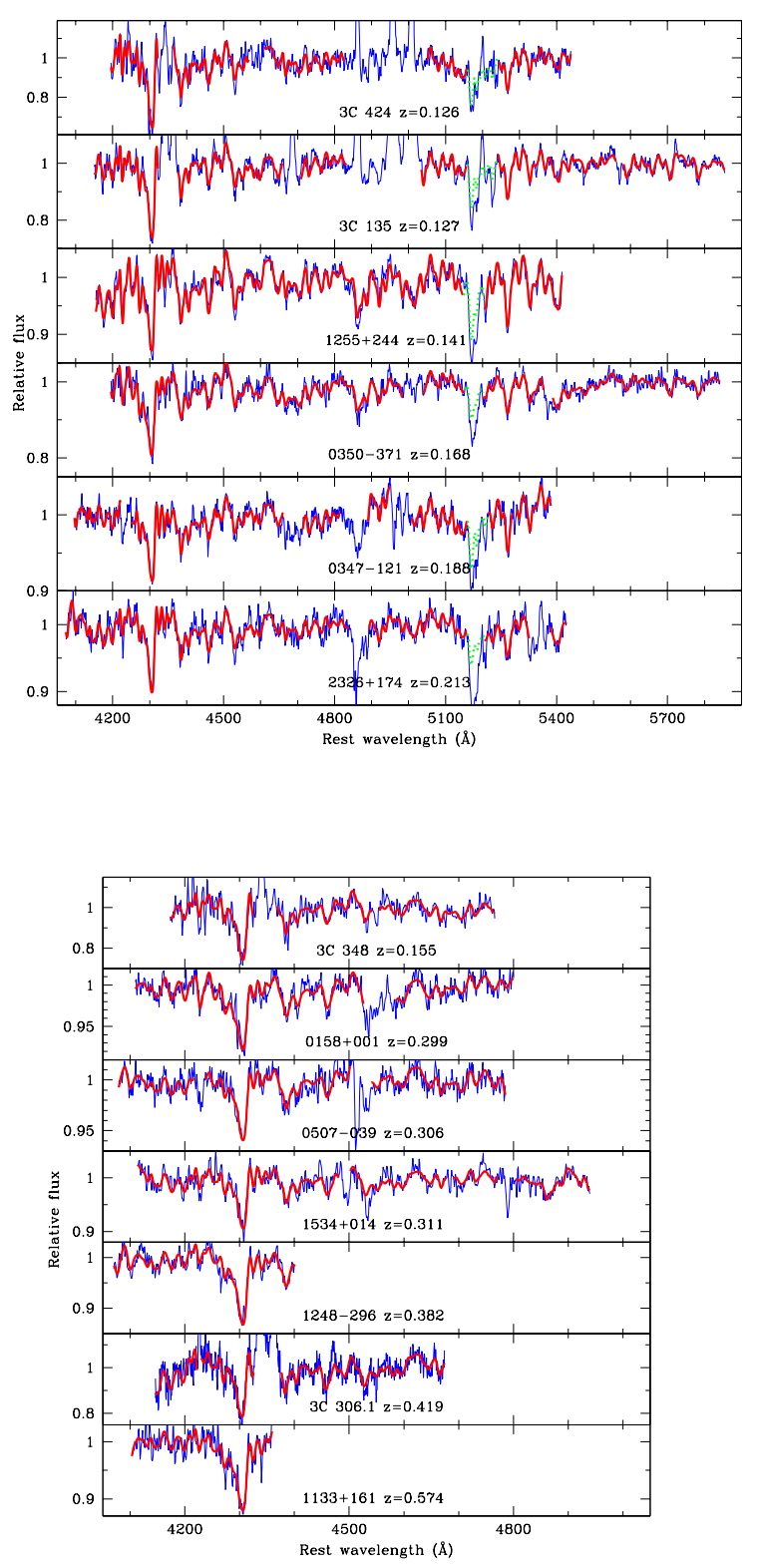

Fig. 1.- Observed spectra of AGN host galaxies (thin line [colored blue in electronic edition]), with best-fit templates (thick line [colored red in electronic edition]). Top panels: Spectra of six galaxies with large wavelength coverage. Bad pixels, AGN emission lines, and the Mgb triplet lines (dotted line [colored green in electronic edition]) were masked out before fitting. Bottom panel: Seven galaxies with smaller fitting ranges.

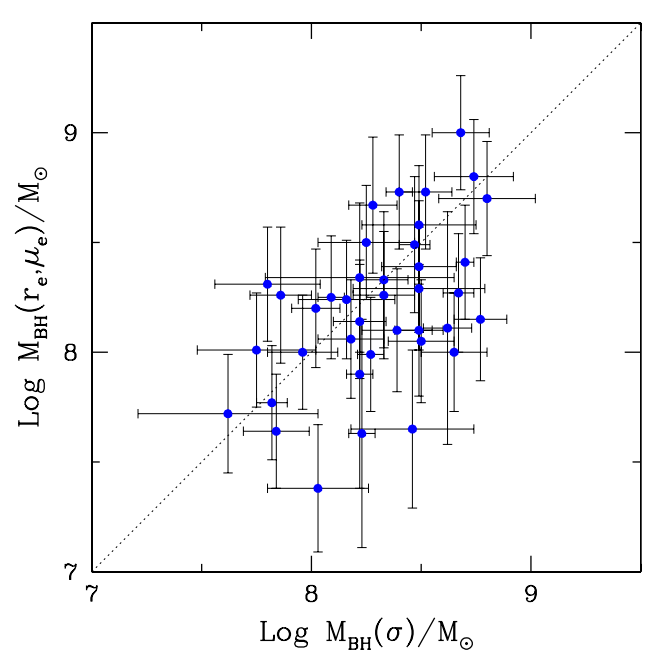

Fig. 2.- Comparison of black hole mass estimates of 32 BL Lac objects from the stellar velocity dispersions (Table 3) and from $r_{e}$ and $\mu_{e}$ using the Coma cluster fundamental plane relation, converted for a cosmology with $H_{o}=$ $70 \mathrm{kms}^{-1} \mathrm{Mpc}^{-1}, \Omega=0.3, \Lambda=0.7$. The surface brightness $\log I$ is corrected for the restframe Cousins $R$-band luminosity evolution of stellar populations using $d \log L / d \log z=0.502$ (see $\S 4.3)$. The measurement errors of $r_{e}, \mu_{e}$, and $\sigma$ are considered in the error propagation. The intrinsic scatter in the fundamental plane $\left(0.08\right.$ in $\left.\log r_{e}\right)$ is also included in the error estimation of black hole mass from $r_{e}$ and $\mu_{e}$ (Woo et al. 2004). The rms scatter between two black hole mass estimates is 0.34 dex. 


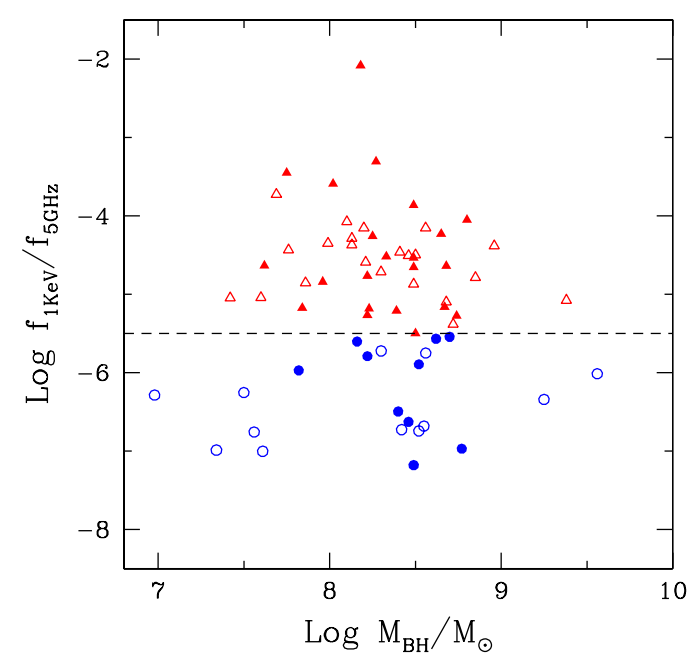

Fig. 3.- X-ray to radio flux ratio vs. black hole mass for our sample of BL Lac objects. Black hole masses are estimated from the measured $\sigma_{e}$ (filled symbols) or $r_{e}$ and $\mu_{e}$ (open symbols). The dashed line divides high-frequency peaked BL Lac objects (triangles) [colored red in electronic edition] from low-frequency peaked objects (circles) [colored blue in electronic edition] following Perlman et al. (1996). The black hole mass spans over 2 orders of magnitude independent of BL Lac spectral type. The black hole mass estimated from $\sigma_{e}$ are distributed over a narrower range because of the relatively small volume sampled $(<z>\sim 0.17)$.

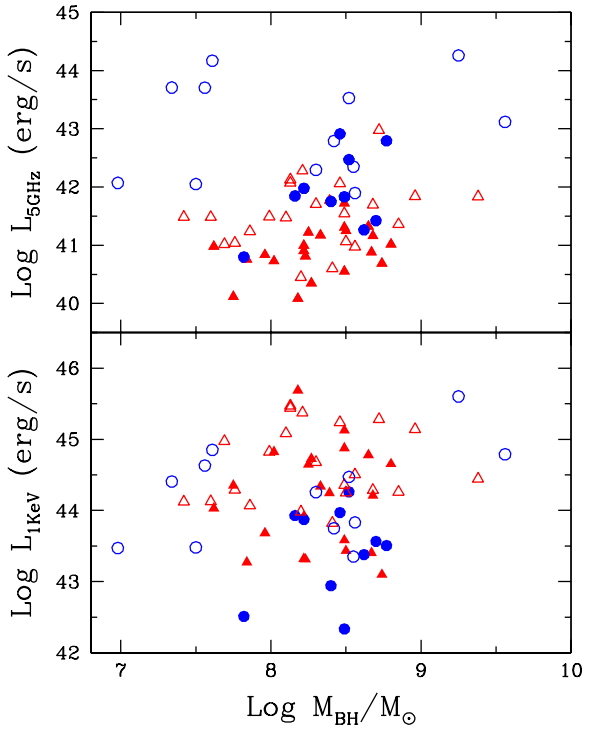

Fig. 4.- Radio and X-ray luminosity vs. black hole mass. Neither X-ray nor radio luminosity is correlated with black hole mass. LBLs tend to have higher radio and lower X-ray luminosity for a given black hole mass. Symbols are the same as in Figure 3. 


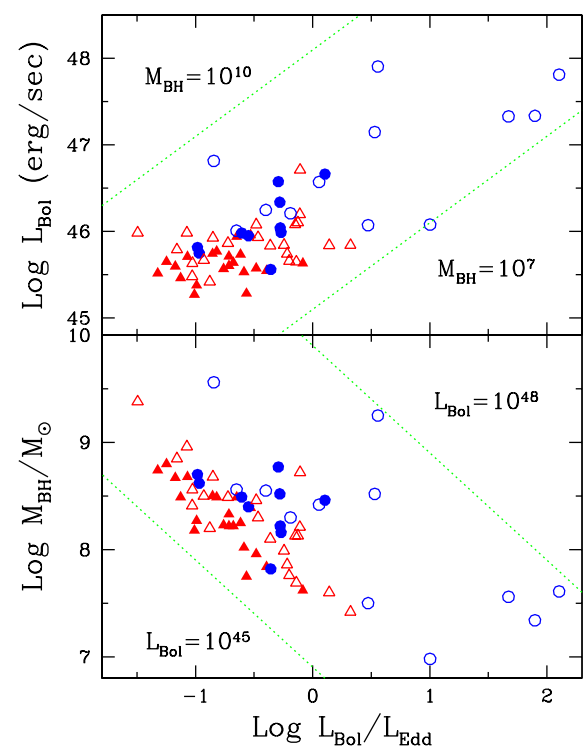

Fig. 5.- Bolometric luminosity (top) and black hole mass (bottom) vs. Eddington ratio. LBLs and HBLs are overlapping in black hole mass. However, LBLs generally have higher Eddington ratios compared with HBLs. Symbols are the same as in Figure 3. The absence of higher and lower black hole mass AGNs in the top panel as well as higher and lower luminosity AGNs in the bottom panel are likely caused by selection effects (see text).

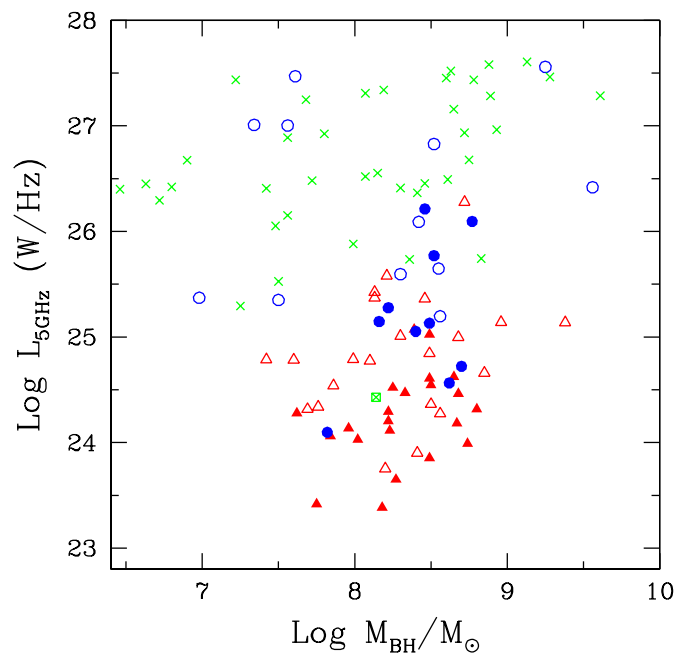

Fig. 6.- Radio luminosity (uncorrected for beaming) of BL Lac objects and FSRQs. The radio luminosity spans over $\sim 4$ orders of magnitude across the mass range of $3 \times 10^{7}<M_{\bullet} / M_{\odot}<10^{9}$. BL Lac objects generally have low radio luminosity compared with FSRQ. FSRQs are represented with crosses (colored in green in electronic edition). Other symbols are the same as in Figure 3. A double-peaked emission line source, PKS 0921213 (cross with a box), shows much lower radio luminosity compared with other FSRQs. 


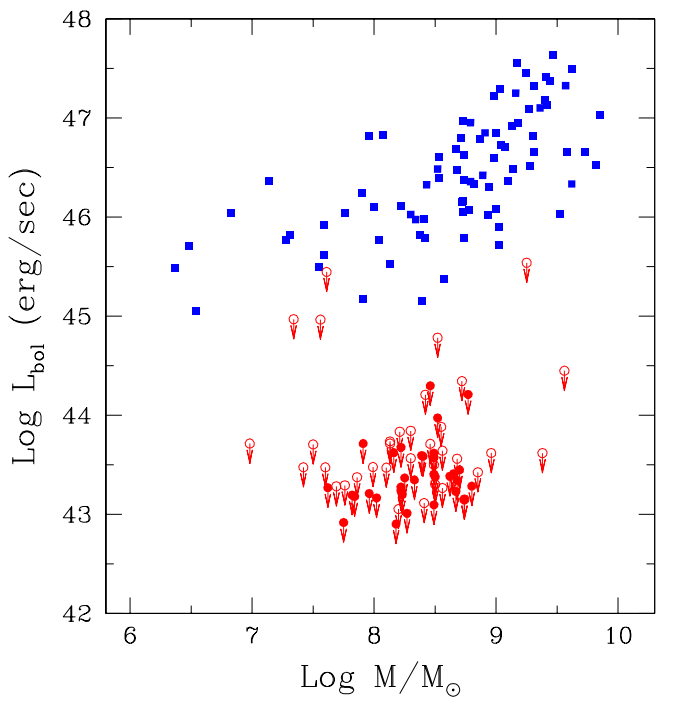

Fig. 7.- Bolometric luminosity vs. black hole mass for radio-loud AGNs. The bolometric luminosities of BL Lac objects are presented as an upper limit, after beaming correction with an averaged lower limit to the Doppler factor, $\delta \gtrsim 3.9$. For the observed black hole mass range $10^{7}<$ $M_{\bullet} / M_{\odot}<4 \times 10^{9}$, the bolometric luminosity spans over 4 orders of magnitude, indicating a large difference in Eddington ratio among radioloud AGNs. Squares: radio-loud AGN from Woo \& Urry (2002a); filled circles: BL Lac objects with black hole mass from the measured $\sigma_{e}$; open circles: BL Lac objects with black hole mass from $r_{e}$ and $\mu_{e}$.

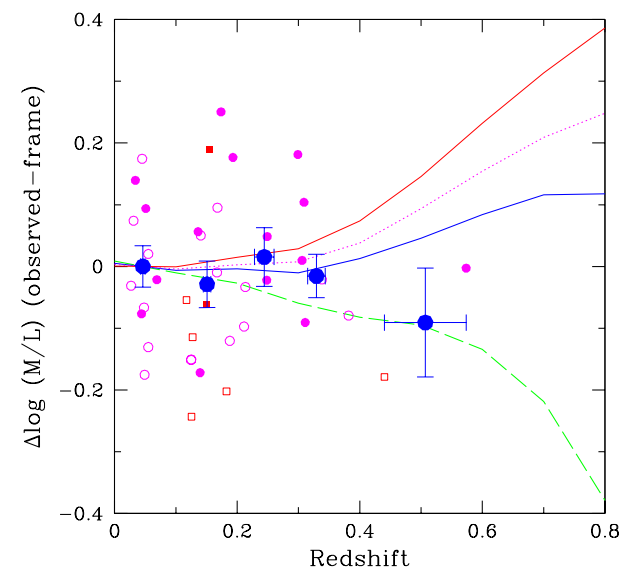

Fig. 8.- Evolution of the observed-frame massto-light ratio of AGN host galaxies in the Cousins $R$ band. The evolution of the mass-to-light ratio is consistent with single burst models with $z_{\text {form }}=1.4_{-0.2}^{+0.9}$ within the $68 \%$ confidence level. $\Delta \log (M / L)$ is defined as the difference in logarithm between the $M / L$ at each redshift and the $M / L$ in our lowest AGN host redshift bin $(z=$ 0.046). Large filled circles: averaged $\Delta \log (M / L)$ for each redshift bin with $1 \sigma$ error bars; small circles: individual host galaxies of BL Lac objects; squares: individual radio galaxies; small open symbols: individual host galaxies with mass less than $5 \times 10^{11} M_{\odot} ;$ small filled symbols: individual host galaxies with mass greater than $5 \times 10^{11} M_{\odot}$; dashed line: stellar population synthesis model with single burst at $z_{\text {form }}=1$; solid line: a single burst model with $z_{\text {form }}=1.5$; dotted line: a single burst model with $z_{\text {form }}=2$; thin-solid line: a single burst model with $z_{\text {form }}=5$. For clarity no error bars are shown on the measurements for individual galaxies. For these the reader is referred to Figure 10, which shows the same measurements with error bars, albeit in the rest-frame rather than the observed frame. 


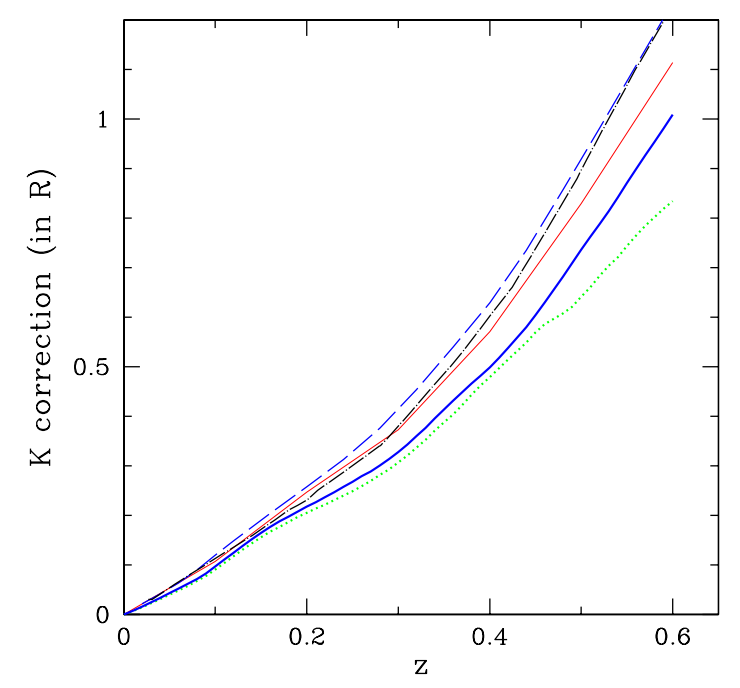

Fig. 9.- $K$-correction values for different stellar population synthesis models. Dotted line: a single burst at $z=1$; solid line: a single burst at $z=1.4$; thin-solid line: a single burst at $z=5$ with a cosmology with $\Omega=0.3, \Lambda=0.7$, and $H_{o}=70 \mathrm{~km}$ $\sec ^{-1} \mathrm{Mpc}^{-1}$. Dashed line: $K$-correction values from Poggianti 1997, where a cosmology with $q_{o}=$ $0.225, H_{o}=50 \mathrm{~km} \mathrm{sec}^{-1} \mathrm{Mpc}^{-1}$ was used. Dotdashed line: $K$-correction values from Fukugita et al. 1995, based on the observed spectra of nearby galaxies with no stellar population evolution using a cosmology with $q_{o}=0$ and $H_{o}=50 \mathrm{~km} \mathrm{sec}^{-1}$ $\mathrm{Mpc}^{-1}$.

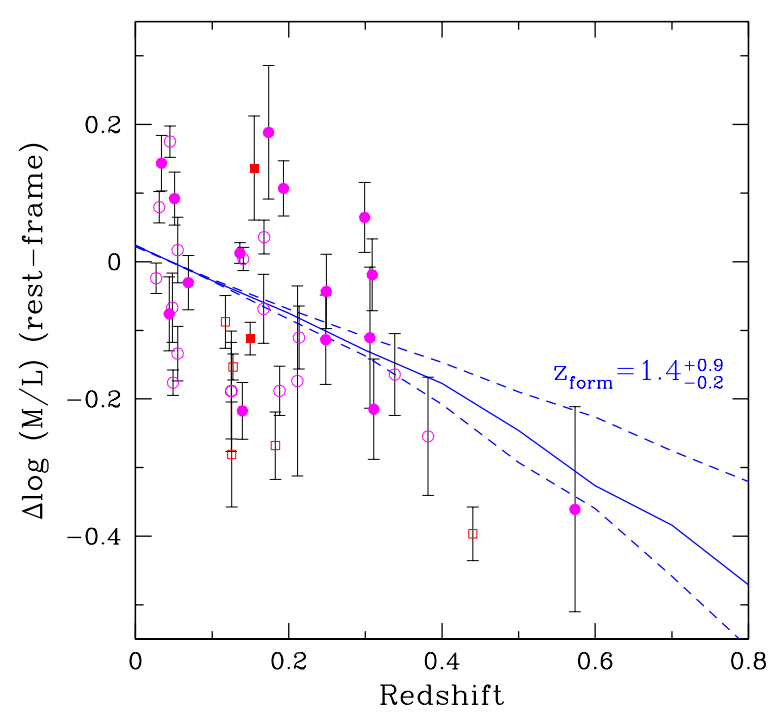

Fig. 10.- Evolution of the rest-frame mass-tolight ratio of AGN host galaxies in the Cousins $R$ band. The mass-to-light ratio for individual galaxies are derived after $K$-correction with the $z_{\text {form }}=1.4$ model. Circles: host galaxies of BL Lac objects; squares: radio galaxies; open sybmols: galaxies with mass $<5 \times 10^{11} M_{\odot}$; filled symbols: galaxies with mass $>5 \times 10^{11} M_{\odot} ;$ solid line: a single burst model with $z_{\text {form }}=1.4$, which has $\Delta \log (M / L) / \Delta z=-0.502$ between $z=0$ and $z=0.4$; dashed lines: single burst models with $z_{\text {form }}=1.2$ and 2.3 , showing the uncertainty range determined in the observed mass-to-light ratio evolution (see §4.2). The observed trend is similar to that of early-type galaxies (Treu et al. 2002; van Dokkum \& Franx 2001; Treu et al. 2002). 


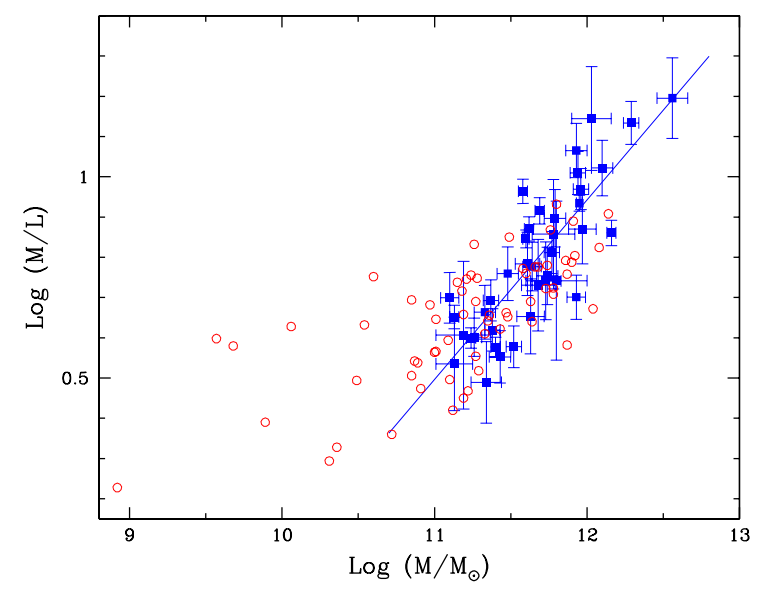

Fig. 11.- Galaxy mass-to-light ratio vs. galaxy mass. The mass-to-light ratios for our AGN host galaxies (filled squares), corrected for luminosity evolution using a $z_{\text {form }}=1.4$ model, are compared with those of nearby elliptical galaxies (open circles) from van der Marel \& van Dokkum (2005). The mass-to-light ratios in the Cousins $R$ band for the nearby galaxies were calculated using $B-R=1.57$. The solid line corresponds to the best fit to the AGN host galaxies. The massto-light ratio vs. mass relation of the AGN host galaxies is consistent with that of normal galaxies in the mass range where the data sets overlap $\left(11<\log M / M_{\odot}<12\right)$. 
TABLE 1

JOURNAL OF OBSERVATIONS

\begin{tabular}{|c|c|c|c|c|c|c|c|c|c|c|}
\hline $\begin{array}{l}\text { Run } \\
\text { (1) }\end{array}$ & $\begin{array}{l}\text { Date } \\
(2)\end{array}$ & $\begin{array}{l}\text { Telescope } \\
\qquad(3)\end{array}$ & $\begin{array}{l}\text { Instrument } \\
\text { (4) }\end{array}$ & $\begin{array}{c}\text { Grating } \\
\text { lines } \mathrm{mm}^{-1} \\
\qquad(5)\end{array}$ & $\begin{array}{l}\text { Slit Width } \\
\text { arcsec } \\
(6)\end{array}$ & $\begin{array}{c}\text { Resolution } \\
\AA \\
(7)\end{array}$ & $\begin{array}{l}\text { Plate Scale } \\
\text { Apixel }^{-1} \\
(8)\end{array}$ & $\begin{array}{l}\text { Spatial Scale } \\
\text { arcsec pixel }^{-1} \\
(9)\end{array}$ & $\begin{array}{l}\text { Seeing } \\
\text { arcsec } \\
(10)\end{array}$ & $\begin{array}{l}\text { Sky } \\
(11)\end{array}$ \\
\hline 1 & $11 / 27 / 03$ & Magellan $6.5 \mathrm{~m}$ & $\mathrm{~B} \& \mathrm{C}$ & 600 & 1 & 2.2 & 1.56 & 0.25 & $0.7-0.9$ & clear \\
\hline 2 & $6 / 14-17 / 04$ & Magellan $6.5 \mathrm{~m}$ & $\mathrm{~B} \& \mathrm{C}$ & 600 & 1 & 2.2 & 1.56 & 0.25 & $0.7-0.9$ & clear \\
\hline 3 & $4 / 20 / 04$ & Gemini-South & GMOS & 400 & 0.5 & 1.4 & 0.69 & 0.07 & $\sim 0.5$ & clear \\
\hline
\end{tabular}

Note.-Col. (1): observing run. Col. (2): observing date. Col. (3): telescope. Col. (4): instrument. Col. (5): grating. Col. (6): slit width. Col. (7): approximate instrumental resolution in Gaussian $\sigma$. Col. (8): plate scale. Col. (9): spatial scale. Col. (10): seeing FWHM from guiding cameras. Col. (11): sky condition. 
TABLE 2

TARGets AND MEASUREMENTS

\begin{tabular}{|c|c|c|c|c|c|c|c|c|c|c|c|c|}
\hline $\begin{array}{c}\text { Name } \\
(1)\end{array}$ & $\begin{array}{l}\mathrm{Z} \\
(2)\end{array}$ & $\begin{array}{c}<\mu_{e}> \\
(3)\end{array}$ & $\begin{array}{l}A_{R} \\
(4)\end{array}$ & $\begin{array}{l}K_{R} \\
(5)\end{array}$ & $\begin{array}{l}m_{R} \\
(6)\end{array}$ & $\begin{array}{c}r_{e} \\
\prime \prime \\
(7)\end{array}$ & $\begin{array}{c}\sigma \\
\mathrm{km} \mathrm{s}_{(8)}^{-1}\end{array}$ & $\begin{array}{l}\mathrm{r} \\
\prime \prime \\
(9)\end{array}$ & $\begin{array}{c}\mathrm{C} \\
(10)\end{array}$ & $\begin{array}{l}\text { Run } \\
\text { (11) }\end{array}$ & $\begin{array}{l}\text { Exp. } \\
\text { hour } \\
(12)\end{array}$ & $\begin{array}{l}\mathrm{S} / \mathrm{N} \\
(13)\end{array}$ \\
\hline $0158+001$ & 0.2991 & 20.13 & 0.06 & 0.327 & 18.27 & $1.90 \pm 0.10$ & $273 . \pm 21$ & 1.25 & 1.08 & 1 & 2 & 116 \\
\hline $0347-121$ & 0.1880 & 19.12 & 0.12 & 0.206 & 17.72 & $1.25 \pm 0.05$ & 188.土 10 & 1.25 & 1.07 & 1 & 1.5 & 122 \\
\hline 0506-039 & 0.3059 & 19.65 & 0.22 & 0.337 & 18.35 & $1.60 \pm 0.13$ & $248 . \pm 39$ & 1.25 & 1.08 & 1 & 1.9 & 120 \\
\hline $1133+161$ & 0.5736 & 19.70 & 0.17 & 0.937 & 19.83 & $1.55 \pm 0.23$ & $212 . \pm 48$ & 1.25 & 1.10 & 2 & 4.3 & 65 \\
\hline $1248-296$ & 0.3819 & 19.00 & 0.20 & 0.469 & 18.87 & $1.10 \pm 0.05$ & $245 . \pm 32$ & 1.25 & 1.09 & 2 & 3.5 & 84 \\
\hline $1255+244$ & 0.1407 & 19.95 & 0.03 & 0.153 & 16.72 & $2.50 \pm 0.05$ & $222 . \pm 6$ & 1.25 & 1.06 & 2 & 1 & 100 \\
\hline $1534+014$ & 0.3110 & 19.99 & 0.15 & 0.345 & 18.16 & $2.00 \pm 0.10$ & $208 . \pm 23$ & 1.25 & 1.08 & 2 & 3 & 85 \\
\hline $2326+174$ & 0.2134 & 19.61 & 0.15 & 0.230 & 17.56 & $1.80 \pm 0.15$ & $228 . \pm 16$ & 1.25 & 1.07 & 2 & 2.5 & 97 \\
\hline 0350-371 & 0.1679 & 19.35 & 0.02 & 0.186 & 17.08 & $1.70 \pm 0.07$ & $276 . \pm 10$ & 1.25 & 1.06 & 2 & 0.8 & 70 \\
\hline $3 \mathrm{C} 135^{a}$ & 0.1274 & 18.99 & 0.31 & 0.135 & 17.05 & $1.52 \pm 0.01$ & $197 . \pm 6$ & 1.25 & 1.05 & 1 & 1 & 76 \\
\hline $3 \mathrm{C} 424^{a}$ & 0.1256 & 19.57 & 0.26 & 0.133 & 16.44 & $2.56 \pm 0.04$ & $171 . \pm 20$ & 1.25 & 1.05 & 2 & 2.8 & 51 \\
\hline $3 \mathrm{C} 348^{b}$ & 0.1549 & 23.39 & 0.25 & 0.171 & 15.64 & $22.96 \pm 0.90$ & $212 . \pm 25$ & 1.25 & 1.06 & 2 & 1.6 & 39 \\
\hline $3 \mathrm{C} 306.1^{a}$ & 0.4403 & 18.69 & 0.27 & 0.581 & 19.36 & $0.90 \pm 0.08$ & $222 . \pm 13$ & 0.58 & 1.06 & 3 & 2.3 & 31 \\
\hline
\end{tabular}

Note. - Col. (1): AGN name. Col. (2): measured redshift Col. (3): average surface brightness within $r_{e}$ in the Cousins $R$ band calculated from total host galaxy magnitude (Urry et al. 2000), using Equation (2) Extinction and $K$-corrected. Col. (4): foreground extinction correction due to our galaxy from Schlegel et al. (1998). Col. (5): $K$-correction from our passive evolution model with $z_{\text {form }}=1.4$. Col. (6): observed host galaxy magnitude in the Cousins $R$ band from Urry et al. (2000). Col. (7): half-light radius and error from Urry et al. (2000). Col. (8): measured stellar velocity dispersion and fitting error of velocity dispersion. Col. (9): extraction radius in arcseconds. Col. (10): correction factor for velocity dispersions to a $3.4^{\prime \prime}$ aperture at the distance of the Coma cluster. Col. (11): observing run. Col. (12): total exposure time in hours. Col. (13): signal-to-noise ratio per pixel, measured at 6000 $\AA$ in each combined galaxy spectrum. The $\mathrm{S} / \mathrm{N}$ ratio is in the observed spectrum, which consists of AGN and galaxy 
emission. Thus, the actual $\mathrm{S} / \mathrm{N}$ ratio for the galaxy absorption lines is much lower.

References. - a) galaxy magnitude, $r_{e}$, and $\left\langle\mu_{e}>\right.$ are from our HST image analysis (see $\left.\S 4.1\right)$. b) galaxy magnitude, $r_{e}$, and $\left\langle\mu_{e}\right\rangle$ are from Roche \& Eales (2000). 
TABLE 3

BlaCK hole MASSES AND hOST GALAXY LUMiNOSITIES

\begin{tabular}{|c|c|c|c|c|c|}
\hline $\begin{array}{l}\text { Name } \\
(1)\end{array}$ & $\begin{array}{l}\text { Type } \\
(2)\end{array}$ & $\begin{array}{c}\mathrm{z} \\
(3)\end{array}$ & $\begin{array}{c}\log \left(M_{\bullet} / M_{\odot}\right) \\
(4)\end{array}$ & $\begin{array}{c}\log \left(M_{G} / M_{\odot}\right) \\
(5)\end{array}$ & $\begin{array}{c}M_{R} \\
(6)\end{array}$ \\
\hline $0122+090$ & $\mathrm{H}$ & 0.3384 & $8.49 \pm 0.17$ & $11.61 \pm 0.08$ & -23.02 \\
\hline $0145+138$ & $\mathrm{H}$ & 0.1250 & $7.75 \pm 0.27$ & $11.13 \pm 0.12$ & -22.16 \\
\hline $0158+001$ & $\mathrm{H}$ & 0.2991 & $8.65 \pm 0.15$ & $11.93 \pm 0.07$ & -23.07 \\
\hline $0229+200$ & $\mathrm{H}$ & 0.1396 & $8.68 \pm 0.13$ & $11.93 \pm 0.06$ & -23.76 \\
\hline 0331-362 & $\mathrm{H}$ & 0.3091 & $8.50 \pm 0.15$ & $12.10 \pm 0.07$ & -23.60 \\
\hline 0347-121 & $\mathrm{H}$ & 0.1880 & $8.02 \pm 0.11$ & $11.26 \pm 0.05$ & -22.42 \\
\hline 0350-371 & $\mathrm{H}$ & 0.1679 & $8.67 \pm 0.07$ & $11.69 \pm 0.03$ & -22.66 \\
\hline 0506-039 & $\mathrm{H}$ & 0.3059 & $8.49 \pm 0.30$ & $11.78 \pm 0.14$ & -23.21 \\
\hline $3 \mathrm{C} 135$ & $\mathrm{R}$ & 0.1274 & $8.09 \pm 0.06$ & $11.24 \pm 0.03$ & -22.27 \\
\hline 0521-365 & $\mathrm{L}$ & $0.055^{a}$ & $8.52 \pm 0.12$ & $11.38 \pm 0.05$ & -22.50 \\
\hline $0525+713$ & $\mathrm{H}$ & 0.2482 & $8.80 \pm 0.22$ & $11.97 \pm 0.09$ & -23.58 \\
\hline 0548-322 & $\mathrm{H}$ & $0.069^{a}$ & $8.22 \pm 0.12$ & $11.77 \pm 0.05$ & -23.00 \\
\hline $0706+591$ & $\mathrm{H}$ & $0.125^{a}$ & $8.25 \pm 0.22$ & $11.63 \pm 0.09$ & -23.13 \\
\hline $0829+046$ & $\mathrm{~L}$ & 0.1737 & $8.46 \pm 0.28$ & $12.03 \pm 0.13$ & -22.95 \\
\hline Mrk421 & $\mathrm{H}$ & $0.031^{a}$ & $8.22 \pm 0.06$ & $11.62 \pm 0.03$ & -22.44 \\
\hline Mrk180 & $\mathrm{H}$ & $0.045^{a}$ & $8.23 \pm 0.06$ & $11.58 \pm 0.03$ & -22.12 \\
\hline MS $1133.7+1618$ & $\mathrm{~L}$ & 0.5736 & $8.22 \pm 0.43$ & $11.80 \pm 0.20$ & -23.90 \\
\hline $1212+078$ & $\mathrm{~L}$ & 0.1363 & $8.70 \pm 0.04$ & $11.95 \pm 0.02$ & -23.23 \\
\hline $1215+013$ & $\mathrm{R}$ & 0.1173 & $8.33 \pm 0.11$ & $11.37 \pm 0.05$ & -22.36 \\
\hline $1215-033$ & $\mathrm{R}$ & 0.1826 & $7.86 \pm 0.14$ & $11.43 \pm 0.07$ & -22.93 \\
\hline 1ES 1248-296 & $\mathrm{H}$ & 0.3819 & $8.49 \pm 0.26$ & $11.68 \pm 0.11$ & -23.37 \\
\hline $1255+244$ & $\mathrm{H}$ & 0.1407 & $8.27 \pm 0.06$ & $11.60 \pm 0.02$ & -22.58 \\
\hline $1342-016$ & $\mathrm{R}$ & 0.1498 & $8.47 \pm 0.07$ & $12.16 \pm 0.03$ & -23.96 \\
\hline 3C306.1 & $\mathrm{R}$ & 0.4403 & $8.28 \pm 0.11$ & $11.52 \pm 0.05$ & -23.42 \\
\hline $1514-241$ & $\mathrm{~L}$ & 0.0490 & $8.40 \pm 0.06$ & $11.40 \pm 0.03$ & -22.65 \\
\hline $1534+014$ & $\mathrm{~L}$ & 0.3110 & $8.16 \pm 0.22$ & $11.73 \pm 0.10$ & -23.38 \\
\hline 3C348 & $\mathrm{R}$ & 0.1549 & $8.03 \pm 0.23$ & $12.56 \pm 0.10$ & -24.13 \\
\hline MRK501 & $\mathrm{L}$ & $0.034^{a}$ & $8.62 \pm 0.11$ & $11.94 \pm 0.05$ & -22.88 \\
\hline 1Zw187 & $\mathrm{H}$ & $0.055^{a}$ & $7.84 \pm 0.15$ & $11.10 \pm 0.06$ & -21.59 \\
\hline 3C371 & $\mathrm{L}$ & $0.051^{a}$ & $8.49 \pm 0.11$ & $11.96 \pm 0.05$ & -23.05 \\
\hline $1853+671$ & $\mathrm{H}$ & 0.2113 & $7.62 \pm 0.41$ & $11.19 \pm 0.18$ & -22.25 \\
\hline $1959+650$ & $\mathrm{H}$ & $0.048^{a}$ & $7.96 \pm 0.16$ & $11.33 \pm 0.07$ & -22.24 \\
\hline $3 \mathrm{C} 424$ & $\mathrm{R}$ & 0.1256 & $7.80 \pm 0.24$ & $11.34 \pm 0.10$ & -22.79 \\
\hline $2143+070$ & $\mathrm{H}$ & 0.2490 & $8.39 \pm 0.16$ & $11.79 \pm 0.07$ & -23.07 \\
\hline $2201+044$ & $\mathrm{~L}$ & $0.027^{a}$ & $7.82 \pm 0.07$ & $11.13 \pm 0.03$ & -21.76 \\
\hline $2254+074$ & $\mathrm{~L}$ & 0.1932 & $8.77 \pm 0.12$ & $12.29 \pm 0.05$ & -23.65 \\
\hline $2326+174$ & $\mathrm{H}$ & 0.2134 & $8.33 \pm 0.14$ & $11.64 \pm 0.06$ & -22.93 \\
\hline $2344+514$ & $\mathrm{H}$ & $0.044^{a}$ & $8.74 \pm 0.18$ & $11.74 \pm 0.07$ & -23.05 \\
\hline 2356-309 & $\mathrm{H}$ & 0.1671 & $8.18 \pm 0.15$ & $11.48 \pm 0.07$ & -22.53 \\
\hline
\end{tabular}


Note. - Col. (1): AGN name. Col. (2): spectral type (L: low frequency peaked BL Lac objects, H: high frequency peaked BL Lac objects, R: radio galaxies), Col. (3): redshift measured from our observations (measurement errors are typically less than 0.0001). Col. (4): black hole mass estimated from $\sigma$, using $M_{\bullet} \propto \sigma^{4.02}$ (Tremaine et al. 2002) and error in black hole mass, derived from $\sigma$ measurement error only. Col. (5): host galaxy mass from Eq. 3 and error. Col. (6): absolute $\mathrm{R}$ magnitude, extinction and $K$-corrected. Mass and magnitude are calculated using $H_{o}=70 \mathrm{kms}^{-1} \mathrm{Mpc}^{-1}$.

References. - a) redshift from Urry et al. (2000). 